\title{
Modalités pour le titrage des solutions employées dans la méthode chimique pour la mesure des débits ${ }^{(*)}$
}

\author{
Processes for titration \\ of solutions used in the chemical method \\ for discharge measurements ${ }^{(*)}$ \\ Modalités d'utilisation du bichromate de sodium \\ Conditions of utilization of sodium bichromate
}

PAR M. DODERO

PROFESSEUR A IA FACUTME DES SCIFNCES DE GMENOHLE

\begin{abstract}
Les avantages du bichromate de sodium: absence dans les eaux naturelles, grande solubilité, très grande sensibilité colorimétrique, facilité d'approvisionnement. - Principe du. dosage colorimétrique. - Mode opératoire proposé : étalonnage d'un photocolorimetre is l'aide d'une série de solutions-témoins de dilur tion connue; lecture directe de la dilution recherchée. - Mode de réalisation des diverses solutions-témoins; limite supérieure de l'erreur.
\end{abstract}

\begin{abstract}
Advantages of sodium bichromate: its absence in natural water, high solubility, very great colorinzetrical sensitivity, and abundant supplies available. Principle of colorimetric titration. Modus operandi proposed: standardization of a colorimetric photometer using a series of test solutions of known dilution; direct reading of the dilution found. Method for making up the various test solutions; upper limit of the error.
\end{abstract}

\section{I. - CARACTÉRISTIQUES D'EMPLOI DU BICHROMATE DE SODIUM}

Le Bichromate de Sodium satisfait à toutes les conditions techniques relatives aux solutions d'injection, énumérées à l'article A. II, dans le fascicule $n^{\circ} 4$ du Code d'Essais des Installations Hydrauliques de la Société Hydrotechnique de France.

Il présente surtout les deux très grands avantages suivants : $1^{\circ}$ Il n'existe pas dans les caux naturelles, ce qui réduit l'expression du débit suivant l'article A. I. du fascicule précité, à :

$$
\mathrm{Q}=q \frac{\mathrm{N}_{1}}{\mathrm{~N}_{2}}
$$

$n$ étant nul.

(*) Cf. la Houille Blanche, $\mathrm{n}^{\circ} 2,1953$, p. 282; $\mathrm{n}^{\circ} 3,1953$, p. 389 et $\mathrm{n}^{\circ} 4,1953$, p. 510. - Des tirés à part de ces articles constitueront un complément au Code d'Essais des Installations Hydranliques de la Société Hydrotechnique de France. 
$2^{\circ}$ Il peut être dosé par méthode colorimétrique.

Cette seconde propriété permet de déduire le débit à mesurer d'un rapport de dilutions sans qu'il soit nécessaire de déterminer le titre exact d'aucun échantillon.

Le rapport $\mathrm{Q} / q=\mathrm{N}_{1} / \mathrm{N}_{2}$ représente la dilution de la solution initiale, ou solution mère, dans l'eau de la rivière.

Si l'on réalise en laboratoire une dilution tris précise $\mathrm{N}_{1} / \mathrm{N}_{2}$, d'un échantillon de la solution mère de concentration $\mathrm{N}_{1}$, égale à la dilution précitée, le rapport du débil de la rivière à celui de I'injection sera égal à cette dilution.

Les opérations reviennent done à réaliser avec la plus grande précision possible une série de dilutions encadrant la dilution réalisée dans la rivière, et de comparer les échantillons ainsi obtenus au moyen d'un photocolorimètre.

L'erreur sur le débit due aux opérations du colorimètre se réduit ainsi à une erreur de dilution qui peut être inférieure à $0,5 \%$ et à l'erreur sur la comparaison de deux échantillons au moyen d'un colorimetre.

Cette simplicité de mise en œuvre de la méthode et la grande précision qu'elle permet constituent un avantage sérieux en faveur de l'emploi du bichromate dont les autres caractéristiques sont d'ailleurs elles aussi intéressantes.
En particulier :

C'est un produit industriel lacile à se procurer (il est utilise en grande quantité pour le tannage des peaux). Il ne présente aucun danger, bien qu'il soit indiqué comme toxique en solution concentrée, son pouvoir colorant énorme constituant une sorte d'auto-protection; en solution diluée, il est sans action sur la vie des poissons.

Sa solubilité est de $66,4 \mathrm{~g}$ pour $100 \mathrm{~g}$ de solution saturée à $30^{\circ}$. Il n'existe pas dans les eaux naturelles, comme déjà indiqué, ce qui, outre la simplification des calculs et des opérations signalés permet d'effectuer la dilution au laboratoire avec de l'eau qui n'est pas celle de la rivière.

La colorimétrie se fait avec précision pour des solutions très diluées, ne renfermant pas plus de $1 \mathrm{mg} /$ litre de solution.

Si la solution mère est concentrée à $100 \mathrm{~g} /$ litre et si la solution finale contient $1 \mathrm{mg}$ /litre comme c'est généralement le cas, on voit qu'un échantillon de solution finale correspond à une dilution de 100.000. Par suite, un débit de 1 litre/s de solution mère suffira pour la mesure d'un débit de $100 \mathrm{~m}^{3} / \mathrm{s}$.

En d'autres termes, un jaugeage exigeant une durée d'injection de 20 minutes nécessitera 1,2 lig de $\mathrm{Cr}^{*} \mathrm{O}^{i} \mathrm{Na}^{2}, 2 \mathrm{H}^{2} \mathrm{O}$ par $\mathrm{m}^{3} / \mathrm{s}$ à mesurer.

\section{II. - POUVOIR COLORANT DU BICHROMATE DE SODIUM}

\section{$1^{\circ}$ Pouvolr colorant Naturel}

Le bichromate de sodium possède un pouvoir colorant énorme.

La couleur jaune qu'il communique à l'eau est encore visible dans les solutions de $5 \mathrm{mg} /$ litre sous $1 \mathrm{~cm}$ d'épaisseur.

En dessous de cette teneur, on peut déceler sa présence dans des solutions incolores en utilisant la diphénylcarbazide comme réactif colorimétrique; la coloration rouge-vineuse obtenue est sensible à l'œil pour des teneurs en chrome de $0,03 \mathrm{mg} / 1\left(0,09 \mathrm{mg} / 1 \mathrm{Cr}^{2} \mathrm{O}^{\top} \mathrm{Na}^{2}, 2 \mathrm{H}^{2} \mathrm{O}\right)$ et les photocolorimètres permettent de révéler $0,002 \mathrm{mg} / 1 \mathrm{de}$ chrome $\left(0,006 \mathrm{mg} / 1 \mathrm{Cr}^{2} \mathrm{O}^{7} \mathrm{Na}^{2}, 2 \mathrm{H}^{2} \mathrm{O}\right)$ en lumière monochromatique $540 \mu$ sous une épaisseur de $1 \mathrm{~cm}$.

\section{$2^{\circ}$ Coloration par ia DIPHÉNyldarbazide}

\section{Préparation du réactif et emploi.}

Réactif à l'acide sulfurique. - La réaction de coloration doit s'effectuer en milieu acide $0,2 \mathrm{~N}$. La quantité d'acide introduite dans le réactif doit etre telle que celte concentration soit atteinte lorsque le réactif sera dams la solution.

On prépare :

A. - Une solution de $0,250 \mathrm{~g}$ de diphénylearbazide dans $100 \mathrm{~cm}^{3}$ d'alcool éthylique à $95 \%$.

B. -- Une solution dacide sulfurique de densité 1,13 ou 17,1 degré $B^{6}$. Liacide sulfurique que l'on emploie doit être dépourvu de pouvoir réducteur; il aura été additionné au préalable de permanganate, jusqu'à l'obtention d'une très légère coloration rose persistante.

On mélange $A$ et $B$ en parties égales.

On utilise $2 \mathrm{~cm}^{\prime \prime}$ de ce réactif $(A+B)$ pour $20 \mathrm{~cm}^{3}$ de solution. La coloration se développe lentement; on a intérêt à tracer à laaide du photocolorimètre la courbe de l'intensité de coloration en fonction du temps, pour connaitre l'instant propice à la mesure où la coloration ne varie plus. L'intensité de coloration dépend de l'orgine de la diphénylcarbazide.

Ce réactif ne se conscrve pas et doit ètre fraichement préparé. 


\section{III. - MODE OPERATOIRE}

Le díbil $Q$ est donné par la relation :

$$
\mathrm{Q}=q \frac{\mathrm{V}_{2}}{\mathrm{~V}_{1}}
$$

$V_{1}$ est le volume d'un échantillon de la solution mère d'injection (quelques centimètres cubes);

$V_{2}$ est le volume de la solution après la dilution dans des ballons jaugés pour avoir une coloration égale à celle de la rivière.

Comme il n'est pas possible d'obtenir facilement cette égalité de teinte, on prépare une série de solutions dont la dilution $V_{2} / V_{1}$ s'exprime par un nombre entier et dont les colorations encadrent la coloration de l'eau de la rivière.

Ces solutions portent le nom de solutions étaIons.

Elles permettent de graduer un colorimetre à cellule photo-électrique en dilution; cette graduation s'effectue en tracant une courbe d'étalonnage où l'on portera en ordonnée par exemple les dilutions $V_{2}$ [ou les inverses $\left.1 /\left(V_{2} / V_{1}\right)\right]$ et en abscisses les divisions $n$ de la graduation du colorimetre correspondant aux indications de l'appareil pour chaque dilution étalon.

Il suffit ensuite de porter l'eau de la riviere dans le photocolorimetre pour comaitre, par l'indication de l'apparcil et le report sur la courbe d'étalomnage, le rapport $V_{2} / V_{1}$ correspondant.

Les opérations seront conduites de façon à obtenir la plus faible erreur possible.

On rappelle que la limite supérieure de l'erreur relative sur le rapport $V_{2} / V_{1}$ est la somme des limites supérieures des erreur's relatives causées par l'opération de dilution et par le photocolorimètre.

$$
\frac{\Delta \frac{V_{2}}{V_{1}}}{\frac{V_{2}}{V_{1}}}=\frac{\Delta_{1} \frac{V_{2}}{V_{1}}}{\frac{V_{2}}{V_{1}}}+\frac{\Delta_{2} \frac{V_{2}}{V_{1}}}{\frac{V_{2}}{V_{1}}}
$$

\section{A. - Opérations de dilution ET LIMITE SUPÉRIEURE DE L'ERREUR. PROCÉDÉ AUX FIOLES JAUGÉES}

Les solutions étalons de dilution connue doivent posséder des colorations encadrant celle de la solution à étudier de dilution inconnue. On peut chercher à les préparer par tâtommement. Il est parfois plus simple de se guider par un calcul.

Le Laboratoire recoit en sénéral un échantillon de la solution mère dont on lui donne le titre (au besoin il vérifie ce titre par analyse chimique); il reçoit également les échantillons de la solution diluée dont le titre est supposé celui que se sont proposé d'atteindre les opérateurs du jaugeage pour obtenir le maximum de précision au photocolorimètre (soit $1 \mathrm{mg} / \mathrm{l}$ a $0,1 \mathrm{mg} / \mathrm{l}$ pour le bichromate de sodium). D'après ces données, il peut calculer, en première approximation, la dilution :

$$
\begin{aligned}
& v t=(v+V) t^{\prime} \\
& \frac{V+v}{v}=\frac{t}{t^{\prime}}=\delta
\end{aligned}
$$

par exemple, si :

$$
\begin{aligned}
t & =12,3 \mathrm{~g} / \mathrm{l} \\
t^{\prime} & =0,0002 \mathrm{~g} / \mathrm{l} \\
v & =1 \mathrm{~cm}^{3} \\
\delta & =\frac{12,3}{0,0002}=61,5 \times 10^{3}
\end{aligned}
$$

A l'aide des ballons jaugés, il prépare ensuite des solutions étalons de dilution $S_{1}, S_{2}, S_{3} \ldots$ exprimées par des nombres entiers encadrant la dilution.

Dans le cas particulier, ces dilutions seront, par exemple:

$$
40.000
$$

\begin{tabular}{|c|c|c|c|c|c|c|c|c|c|}
\hline Capacité cm: & 10 & 25 & 50 & 100 & 200 & 250 & 500 & $1.000 \mid$ & 2.000 \\
\hline \multirow{2}{*}{$\begin{array}{l}\text { Tolé- } \\
\text { rance } \\
\text { en : }\end{array}$} & 0,015 & 0,03 & 0,05 & 0,10 & 0,12 & 0,15 & 0,25 & 0,30 & 0,50 \\
\hline & 0,15 & 0,12 & 0,10 & 0,10 & 0,06 & 0,06 & 0,05 & 0,03 & 0,025 \\
\hline
\end{tabular}

100.000

La précision avec laquelle ces dilutions seront faites sera fonction du choix des ballons jaugés.

On peut disposer de ballons jaugés de précision :

Floles JAvgress DE paŕcision. Norme française B 35.027 
Exemple :

Préparation de la dilution 50.000

1. On prendra $10 \mathrm{~cm}$ " de la solution mère, mesurés avec le ballon jaugé (précision $0,015 / 10$ ) et on étendra à $1.000 \mathrm{~cm}^{: 3}$ (précision $0,3 / 1.000$ ).

Dilution obtenue : $\frac{1.000}{10}=100$.

Précision : $\frac{1,5}{1.000}+\frac{0,3}{1.000}=\frac{1,8}{1.000}$

2" On répétera cette mème opération :

Dilution obtenue : 100.

Précision : $\frac{1,8}{1.000}$

3" On prendra $100 \mathrm{~cm}^{*}$ de cette solution (précision $(0,1 / 100)=(1 / 1.000)$ que l'on étendra à 500 (précision $0,25 / 500=0,5 / 1.000$ ).

Dilution obtenue : 5 .

$$
\text { Précision : } \frac{1}{1.000}+\frac{0,5}{1.000}=\frac{1,5}{1.000}
$$

Dilution totale : $100 \times 100 \times 5=50.000$.

Précision :

$\frac{1,8}{1.000}+\frac{1,8}{1.000}+\frac{1,5}{1.000}=\frac{5,1}{1.000}=\frac{0,5}{100}$

Préparation de la dilution 80.000

$1^{\circ}$ On étendra $10 \mathrm{~cm}^{3}$ de la solution mère à $2.000 \mathrm{~cm}^{3}$.

Dilution obtenue : 200 .

Précision :

$$
\frac{1,5}{1.000}+\frac{0,25}{1.000}=\frac{1,75}{1.000}
$$

2" On étendra $10 \mathrm{~cm}^{3}$ de cette solution à 2.000

Dilution obtenue : 200 .

Précision : $\frac{1,75}{1.000}$

$3^{\circ}$ On étendra $1.000 \mathrm{~cm}^{3}$ de cette dernière solution à $2.000 \mathrm{~cm}^{3}$.

Dilution obtenue : 2.

Précision :

$$
\frac{0,3}{1.000}+\frac{0,25}{1.000}=\frac{0,55}{1.000}
$$

Dilution totale : $200 \times 200 \times 2=80.000$.
Précision :

$$
\frac{1,75}{1.000}+\frac{1,75}{1.000}+\frac{0,55}{1.000}=\frac{4,05}{1.000}=\frac{0,4}{100}
$$

Procédé à la burette:

La série normalisée des flacons jaugés ne permet pas d'effectuer toutes les dilutions. Pour remédier à cet inconvénient, on pourra utiliser les burettes employées en analyse volumétrique qui, par ailleurs, sont plus commodes mais moins précises.

Les burettes de précision dont on dispose possèdent habituellement les graduations suivantes:

Burettes de $2 \mathrm{~cm}^{3}$ : graduées au $1 / 100$.

Burettes de $10 \mathrm{~cm}^{3}$ : graduées au $1 / 20$.

Burettes de $50 \mathrm{~cm}^{3}$ : graduces au $1 / 5$.

\section{Exemple :}

1"Au moyen dë la burette de $2 \mathrm{~cm}^{*}$, on prélève un volume de $1,67 \mathrm{~cm}^{3}$ de la solution mère que l'on étend à $2.000 \mathrm{~cm}^{3}$ dans une fiole jaugée.

Dilution obtenue : $\frac{2.000}{1,67}$

Précision : $\frac{1}{1,67}+\frac{2,5}{10.000} \# 0,6 \%$.

$2^{\circ}$ On prélève $10 \mathrm{~cm}^{3}$ de cette solution que l'on étend à $500 \mathrm{~cm}^{3}$.

Dilution obtenue: $\frac{500}{10}$

Dilution totale $: \frac{2.000}{1,67} \times \frac{500}{10}=60.000$.

Précision :

$\frac{1}{1,67}+\frac{2,5}{10.000}+\frac{15}{10.000}+\frac{5}{10.000} \# 0,82 \%$

\begin{tabular}{|c|c|c|c|c|}
\hline Pipettes en $\mathrm{cm}^{3}$ & 1 & 2 & 5 & 10 \\
\hline \multirow{2}{*}{$\begin{array}{c}\text { Tolérance } \\
\text { en : }\end{array}$} & $\pm 0,01$ & $\pm 0,015$ & $\pm 0,015$ & $\pm 0,02$ \\
\hline & \pm 1 & $\pm 0,75$ & $\pm 0,3$ & $\pm 0,2$ \\
\hline Pipettes en $\mathrm{cm}^{3}$ & 20 & 25 & 50 & 100 \\
\hline \multirow{2}{*}{$\begin{array}{c}\text { Tolérance } \\
\text { en : }\end{array}$} & $\pm 0,03$ & $\pm 0,03$ & $\pm 0,04$ & $\pm 0,06$ \\
\hline & $\pm 0,15$ & $\pm 0,12$ & $\pm 0,08$ & $\pm 0,06$ \\
\hline
\end{tabular}

Dans certains cas, on peut améliorer la précision en utilisant les pipettes normalisées.

Nonmes : B 35.024 et B 35.025 
Mode d'emploi des pipelles:

L'affeurement a lieu par écoulement, la pipette étant tenue verticalement, sa pointe appuyée contre la paroi du vase; le vase étant très légèrement incliné, on laisse écouler entièrement et librement le liquide. La pipette est laissée dans la position de vidange 3 secondes après cessation de l'écoulement visible, puis retirée dri vase.

B) Opération Colorimétrigue ET LIMITE SUPĹRIEURE DE L'ERREUR

Le Bichromate de Sodium, en solution très diluée, aux concentrations de l'ordre de $1 \mathrm{mg} / \mathrm{l}$, suit la loi de BeEr. Lorsqu'on utilise un photocolorimètre qui répond linéairement à la densité optique, la courbe de l'inverse du rapport:

$$
\frac{V_{2}}{V_{1}}
$$

c'est-à-dire : $\quad \frac{1}{\frac{V_{2}}{V_{1}}}=\frac{V_{1}}{V_{2}}$

en fonction de la graduation $n$ du colorimètre est une droite.

$$
\frac{V_{1}}{V_{2}}=k n
$$

ou :

$$
\frac{V_{2}}{V_{1}}=\frac{1}{k n}
$$

$$
\frac{\Delta \frac{V_{2}}{V_{1}}}{\Delta n}=-\frac{1}{k n^{2}} \quad \text { et } \frac{\Delta \frac{V_{2}}{V_{1}}}{\frac{V_{2}}{V_{1}}}=-\frac{\Delta n}{n}
$$

$\Delta n$ est la limite supérieure de l'erreur absolue de l'indication $n$ de l'appareil. Si l'appareil était parfaitement stable et les mesures parfaitement reproductibles, $\Delta n$ se raménerait à l'erreur de lecture qui peut ètre, suivant la graduation, de $1 / 4$ ou $1 / 2$ division.

Mais, en général, l'appareil n'est pas parfaitement fidèle.

Son manque de fidélité peut être caractérisé par l'écart quadratique moyen d'une série d'observations d'un même échantillon.

Dans le cas de très faibles concentrations finales, il y a lieu de vérifier qu'aucune erreur systématique ne peut être introduite du fait qu'une dilution du bichromate de mème valeur effectuée dans la rivière ou en laboratoire peut conduire à des indications différentes $\mathrm{du}$ colorimètre (collö̈des, matières dissoutes, adsorption du bichromate, etc.).

\section{Mode d'emploi du photocolorimètre:}

On se conformera strictement aux instructions données par le constructeur de l'appareil. Ces instructions varient avec le type d'appareil. Cependant, on aura soin de surveiller avec la plus grande précaution la stabilité du réseau d'alimentation d'énergie électrique dont la tension doit rester rigomreusement constante. Il sera le plus souvent nécessaire d'utiliser un stabilisateur de tension.

\section{IV. - CONDUITE D'UNE OPÉRATION COMPLÈTE DE DÉTERMINATION DU RAPPORT $V_{2} / V_{1}$}

On prépare les solutions étalons comme il a été dit précédemment en utilisant l'eau de la rivière si possible et on trace la courbe d'étalonnage.

Les échantillons d'eau prélevés dans la rivière sont recus dans des flacons spéciaux en verre munis d'une fermeture en caoutchouc. Ils sont numérotés et rangés par ordre.

Autant de béchers de $50 \mathrm{~cm}^{3}$ portant les mêmes numéros que les flacons sont disposés sur la table du laboratoire. On y introduit $20 \mathrm{~cm}^{3}$ de l'échantillon avec une pipette de précision.

A l'aide d'une pipette de $2 \mathrm{~cm}^{3}$, on introduit $2 \mathrm{~cm}^{3}$ du réactif à la diphénylcarbazide. On agite pour obtenir un mélange parfait. On attend que la coloration ait évolué. On prélève la quantité de liquide nécessaire pour remplir la cuve du photocolorimètre et on effectue les mesures au bout d'un temps déterminé, toujours le même, après l'introduction du réactif dans chaque echantillon (10 à 15 minutes par exemple). Cette précaution est nécessaire pour que les mesures restent comparables d'un échantillon à l'autre dans le cas où la coloration serait encore en cours d'évolution.

Pour éviter d'attendre entre les mesures successives, on pourra synchroniser les introductions du réactif dans les échantillons avec les 
lectures sur le pholocolorimètre avec un décalage de temps constant.

Nota. - - On s'assurera que toutes les précautions relatives aux mesures colorimétriques anront été prises, en particulier en ce qui concerne la propreté des flacons d'échantillonnage. Si l'eau de la rivière contient des poussières en suspension et si la dilution en ballons jaugés se fait au laboratoire avec de l'eau pure, il faut décanter ou centrifuger l'eau de la rivière ou la filtrer avant la mesure. Il est recommandé de procéder à la filtration des le flaconnage, au moment du prélévement, dans le cas où les maféraux en suspension auraient une action sur la solution. Suivant A. GunTz, Professeur à la Faculté d'Alger - (CRAS, tome 236, p. 24232424, séance du 22 juin 1953) - des microorganismes divers sont susceptibles de réduire le bichromate en milieu neutre ou alcalin; pour rendre applicable aux eaux contenant ces microorganismes la méthode au chromate, on peut stopper le processus de réduction par l'introduction du permanganate des be prólevement de l'echantillon.
Matériel nécessaire :

1 photocolorimetre;

2 ou 3 séries de pipettes normalisées;

- 2 ou 3 séries de ballons jaugés normalisés;

- 1 ou 2 burettes de précision de 2, de 10 et de $50 \mathrm{~cm}^{3}$;

- 50 ou 100 béchers de $50 \mathrm{~cm}^{3}$;

- Quelques béchers de 100 ou $200 \mathrm{~cm}^{*}$.

Quelques auteurs a consulter sur la colorimétrie :

SAXDELL. - Colorimetric determination of traces of metals, vol. III, Chemical analysis, Interseience, NewYork, 1944.

Charrot. -.. Methodes modernes d'analyse quantilative minérale, Masson, 1949.

Charlot et Gavgun. - Dosages colorimétriques, Masson, 1952.

B. Lange. - Analyse colorimétrique, Dunod, 1947.

Sxrr.t. - Colorimetric methods of a alysis, D. van Nostrand Cy, New-York, 1949.

\section{RECOMMANDATIONS...}

Vous vous êtes procuré ce numéro parce que l'un de ses articles coïncide avec vos préoccupations actuelles. Les autres articles, nos autres numéros, vous semblent sans utilité pratique.

Mais savez-vous quelles seront vos préoccupations, dans quelques années, dans quelques mois même? Le numéro que vous jugez aujourd'hui sans intérêt vous apparaîtra alors comme un instrument de travail absolument indispensable. Vous nous le demanderez et - ceci se produit journellement - nous vous répondrons qu'il est épuisé.

ABONNEZ-VOUS; feuilletez chaque numéro au moment où il vous parvient. Les articles présentés ne vous sont peut-être d'aucun secours aujourd'hui, mais, demain, vous saurez qu'ils existent, et lorsque vous en aurez besoin vous les retrouverez sans la moindre difficulté dans VOTRE collection.

ABONNEZ-VOUS; c'est plus sûr, plus commode et beaucoup moins cher.

LA HOUILLE BLANCHE. 